\title{
Gene expression differences between stroke-associated and asymptomatic carotid plaques
}

\author{
Jani Saksi • Petra Ijäs • Krista Nuotio • Riitta Sonninen • Lauri Soinne • Oili Salonen • \\ Eija Saimanen • Jarno Tuimala • Erno M. Lehtonen-Smeds • Markku Kaste • \\ Petri T. Kovanen $\cdot$ Perttu J. Lindsberg
}

Received: 21 January 2011 /Revised: 6 May 2011 /Accepted: 11 May 2011 /Published online: 24 May 2011

(C) The Author(s) 2011. This article is published with open access at Springerlink.com

\begin{abstract}
Atherosclerotic carotid stenosis is an important risk factor for stroke. Carotid plaques (CPs) causing stroke may present a distinct type of molecular pathology compared with transient ischemic attack (TIA)-associated or asymptomatic plaques. We compared the gene expression profiles of CPs from stroke patients $(n=12)$ and asymptomatic patients $(n=9)$, both with similar risk factors and severity of carotid stenosis $(>70 \%)$. Sixty probes showed over 1.5 -fold expression difference at $5 \%$ false
\end{abstract}

Jani Saksi and Petra Ijäs contributed equally to this article

Electronic supplementary material The online version of this article (doi:10.1007/s00109-011-0773-z) contains supplementary material, which is available to authorized users.

J. Saksi $(\bowtie) \cdot$ P. Ijäs $\cdot$ K. Nuotio $\cdot$ R. Sonninen · P. J. Lindsberg

Research Programs Unit, Molecular Neurology,

Biomedicum Helsinki, University of Helsinki,

P.O. Box 700, Haartmaninkatu 8,

FI-00290, Helsinki, Finland

e-mail: jani.saksi@helsinki.fi

P. Ijäs $\cdot$ K. Nuotio $\cdot$ L. Soinne $\cdot$ M. Kaste $\cdot$ P. J. Lindsberg

Department of Neurology, Helsinki University Central Hospital,

Helsinki, Finland

O. Salonen

Department of Radiology, Helsinki University Central Hospital, Helsinki, Finland

E. Saimanen

South Karelia Central Hospital,

Lappeenranta, Finland

J. Tuimala

CSC-Scientific Computing Ltd.,

Espoo, Finland

E. M. Lehtonen-Smeds $\cdot$ P. T. Kovanen

Wihuri Research Institute,

Helsinki, Finland discovery rate. Functional clustering showed enrichment of genes in $51 \mathrm{GO}$ categories and seven pathways, the most significant of which relate to extracellular-matrix interaction, PPAR gamma signaling, scavanger receptor activity, and lysosomal activity. Differential expression of ten genes was confirmed in an extended replication group $(n=43)$, where the most significant expression differences were found in CD36 (2.1-fold change, $p=0.005$ ), CD163 (1.7fold change, $p=0.007)$ and FABP4 (2.2-fold change, $p=$ $0.015)$. These include four genes not previously linked to plaque destabilization: GLUL (2.2-fold change, $p=0.016$ ), FUCA1 (2.2-fold change, $p=0.025)$, IL1RN (1.6-fold change, $p=0.034$ ), and S100A8 (2.5-fold change, $p=$ 0.047). Strong correlations were found to plaque ulceration, plaque hemorrhage, and markers of apoptosis and proliferation (activated caspase 3, TUNEL, and Ki67). Protein expression of these genes was confirmed by immunohistochemistry and was found in the atheromatous areas of CPs critical for plaque destabilization. This study presents a comprehensive transcriptional analysis of stroke-associated CPs and demonstrates a significant transcriptome difference between stroke-associated and asymptomatic CPs. Followup studies on the identified genes are needed to define whether they could be used as biomarkers of symptomatic CPs or have a role in plaque destabilization.

Keywords Stroke - Atherosclerosis · Microarray · Cerebrovascular $\cdot$ Real-time PCR

\section{Introduction}

Atherosclerotic disease of the internal carotid artery is a major risk factor of ischemic stroke, and $20 \%$ of patients with anterior and middle cerebral artery infarcts have significant 
carotid stenoses. Curiously, carotid stenoses behave very differently; some progress to occlusion without causing any symptoms, while others manifest themselves with a sudden massive atherothromboembolic cerebral infarction. Previous ipsilateral symptoms and stenosis of $70 \%$ or more are established predictive factors of future ipsilateral stroke [1, 2]. Several histological features are also more frequent in symptomatic carotid plaques (CPs), especially fibrous cap thinning, greater inflammatory cell infiltrations, intraplaque hemorrhage, and plaque rupture [3-6]. Both radiologically and histologically detected ulcerations have been shown to associate with symptoms $[3,5,7]$. The detailed molecular mechanisms underlying these plaque features and $\mathrm{CP}$ destabilization are incompletely understood.

Several studies have employed large-scale gene expression analysis as a tool to find novel pathophysiologically important genes or signaling pathways that could trigger CP destabilization. Vemuganti and Dempsey conducted a whole genome microarray analysis of CPs from six symptomatic and four asymptomatic patients and reported 289 differentially expressed genes [8], the significance of which, however, is difficult to evaluate because of the lack of adequate statistical tests or replication. In a slightly different approach, Papaspyridonos and colleagues compared unstable and stable plaque regions from three patients and identified a large number of differentially expressed genes in both intra- and inter-individual comparisons [9]. Twenty-seven of these were replicated in an independent set of 46 unstable and stable plaque regions, and matrix metalloproteinase-9 (MMP-9), cathepsin B, and legumain further at the protein level. Dahl and colleagues compared expression patterns in four symptomatic and four asymptomatic CPs and found 136 genes with more than twofold expression change, none of which were statistically significant [10]. In subsequent studies in a replication group of $21 \mathrm{CPs}$, they showed one of these genes, visfatin, to be significantly over-expressed both at the RNA and protein level in symptomatic CPs as well as strong protein expression at the site of plaque rupture in acute coronary syndrome patients. Recently, Agardh et al. compared the expression profiles of CPs from patients with recent symptom ( $\leq 1$ month; $n=14$ ), patients with longer delay $(>1$ month; $n=17)$, and asymptomatic patients $(n=9)$ and showed the over-expression of fatty acid-binding protein 4 (FABP4) in CPs from patients with recent symptoms compared to asymptomatic patients [11]. In addition to FABP4, they report two other genes, IQ motif containing GTPase-activating protein 1 and $\mathrm{CD} 84$ antigen, to be upregulated in symptomatic CPs. Also proteomics approaches utilizing a protein array of 512 antibodies [12] and two-dimensional gel electrophoresis in connection with mass spectrometry [13] have been used to compare protein expression in stable and unstable carotid plaques. Despite of a number of studies done, some limiting issues impede drawing firm conclusions on the gene expression changes typical of stroke-associated CPs: the very small number of samples in some studies, diverse clinical diagnoses of patients (amaurosis fugax, TIA, or stroke) and the small number of findings reaching statistical significance.

Previously, we have carried out a microarray study on rare cases of bilateral carotid stenosis when searching for intra-individual local factors influencing plaque stability and identified genes involved in the clearance of free tissue hemoglobin, CD163 molecule (CD163), and heme oxygenase-1, to be important [14]. Now we report a larger microarray study of two extreme patient groups with similar conventional risk factors and severity of carotid stenoses but dissimilar clinical plaque behavior to identify interindividual differences. Due to the inherent inaccuracy in the diagnosis of temporary symptoms such as in TIA and recent findings suggesting that TIA or stroke may represent distinct plaque pathologies [5, 6], we restricted our analysis to stroke-associated carotid plaques (SCPs) from patients with imaging-confirmed recent ipsilateral ischemic brain infarct and asymptomatic carotid plaques (ACPs) from patients without cerebrovascular symptoms and normal brain imaging.

\section{Materials and methods}

\section{Patients}

The patients were selected from the Helsinki Carotid Endarterectomy Study (HeCES) cohort, which included 92 patients who underwent carotid endarterectomy due to high-grade carotid stenoses (>70\% [1]) during 1997-2000 in the Helsinki University Central Hospital (methods in detail [15-17]). Recruitment was in-group consecutive, i.e., the recruitment of asymptomatic patients continued 2 months longer to get a more balanced cohort symptomwise. All patients underwent thorough clinical examination and interview by a stroke neurologist, DSA angiography, transcranial Doppler ultrasound, brain imaging (CT or MRI), routine blood analysis, and analysis of serum lipids and fibrinolytic system. The clinical data was gathered in a HeCES databank together with plaque-level histological and gene expression data. The division into symptomatic or asymptomatic CPs was based on the presence or absence of prior ipsilateral ischemic cerebral or retinal symptoms within 120 days prior to surgery. All human studies were performed in accordance with the Declaration of Helsinki and informed consent was obtained from all patients. The study protocol was approved by the University of Helsinki ethics committee of ophtalmology, otorhinolaryngology, neurology, and neurosurgery (\#339/E9/04). 
This microarray study includes CPs only from symptomatic patients with clinical stroke and radiologically confirmed ischemic brain infarction in the ipsilateral carotid territory $(n=12)$ and asymptomatic patients with no history of ischemic cerebral or retinal symptoms and normal brain imaging $(n=9)$. In addition, we performed quantitative real-time RT-PCR in an extended group of patients with a clinically defined ischemic stroke or retinal infarct in the ipsilateral carotid territory $(n=25)$ and clinically asymptomatic patients $(n=18)$. This group of patients $(n=43)$ is referred to as the "extended replication group" in the text. There is a significant gender difference between the stroke and asymptomatic patients in both the microarray $(p=0.023)$ and the extended replication $(p=0.055)$ groups. Patient characteristics are given in Table 1.

\section{Sample preparation}

The CPs were removed en bloc in endarterectomy. The vascular surgeon (ES) inspected CPs after gentle wash of saline, and made macroscopic observations about surface ulceration, intraplaque hemorrhage, intramural thrombus, loose atheroma, and calcification. The plaques were cut into five longitudinal slices, all containing a portion of atheroma core, each of which were used for a specific purpose: histological examination, RNA and protein extraction, and biochemical analyses. Total RNA was extracted with Trizol $^{\circledR}$-reagent (Invitrogen Life Technologies) and purified with an RNeasy Total RNA Isolation Kit (Qiagen) according to the manufacturers' recommendations. RNA quality was assessed using the RNA 6000 Nano LabChip Kit (Agilent Bioanalyser 2100; Agilent Technologies). Details on RNA quality assessment can be found in Supplemental Material.

\section{Microarray analysis and quantitative real-time RT-PCR}

The microarray experiments were performed according to the manufacturer's recommendations and the MIAME guidelines [18] using Affymetrix GeneChip ${ }^{\circledR}$ U133A arrays containing 22,283 probe sets. The flowchart of the microarray data analysis is shown in Fig. 1. Detailed description on analysis steps can be found in Supplemental Material.

Quantitative real-time RT-PCR (qPCR) was performed using TaqMan ${ }^{\circledR}$ Gene Expression Assays and the ABI PRISM $^{\circledR} 7000$ Sequence Detection System (Applied Biosystems) according to the manufacturer's recommendations. Beta-actin was used as the endogenous control gene and relative quantitation was performed using either the comparative delta-delta- $\mathrm{Ct}$ or the relative standard curve method. For details see the Supplemental Material and Supplemental Table 1.
Immunohistochemistry and microscopy

Microscopic analyses were performed in adjacent segments of the plaques used for RNA extraction. CPs were assigned to AHA-classes based on microscopic histology [19]. The amounts of macrophages, activated $\mathrm{T}$ cells, mast cells, and smooth muscle cells were determined by immunohistochemistry [16]. In addition, the protein products of the following genes were immunostained in the microarray group either in fresh frozen or paraffin-embedded sections using methods previously described $[14,15,20]$ : CD36 molecule (CD36), perilipin 2 (PLIN2), FABP4, glutamateammonia ligase (GLUL), chemokine (C-C motif) ligand 18 (CCL18), interleukin 1 receptor antagonist (IL1RN), heme oxygenase 1 (HMOX1), and S100 calcium binding protein A8 (S100A8; Supplemental Table 2). Light microscopy was performed using Axioplan 2, AxioCam color, and MRGrab 1.0.0.4 respectively (all from Carl Zeiss) by one investigator $(\mathrm{KN})$, who was blinded to the clinical data. For details see Supplemental Material.

\section{Statistics}

The statistical analyses, apart from the microarray analyses, were performed using SPSS10.0.7 for Windows (SPSS Inc.). Pearson's chi square or Fisher's exact test was used to evaluate the differences in non-continuous variables, and the independent-samples $t$ test was employed for continuous variables. The results from qPCR were evaluated by the Mann-Whitney $U$ test. Correlations between real-time qPCR results and clinical data and plaque characteristics were calculated by nonparametric Spearman's Rank Order Correlation Coefficient with Benjamini-Hoechberg multiple testing correction.

\section{Results}

Macroscopic and histological characterization of CPs

We first explored macroscopic and histological differences between SCPs and ACPs. There were no differences in macroscopic intraplaque hemorrhages, intramural thrombus, loose atheroma, or calcification. The SCPs had more macroscopic ulcerations (Table 1). On microscopic examination, all CPs represented complicated AHA class VI lesions containing microscopic surface ulceration (VIa), hematoma/hemorrhage (VIb), and/or thrombotic deposit (VIc, see Supplemental Figure 3) [19]. No significant quantitative differences were found in the immunostained area for macrophages, activated T cells, mast cell numbers, or smooth muscle cell staining grades (Supplemental Figures 4 and 5). 


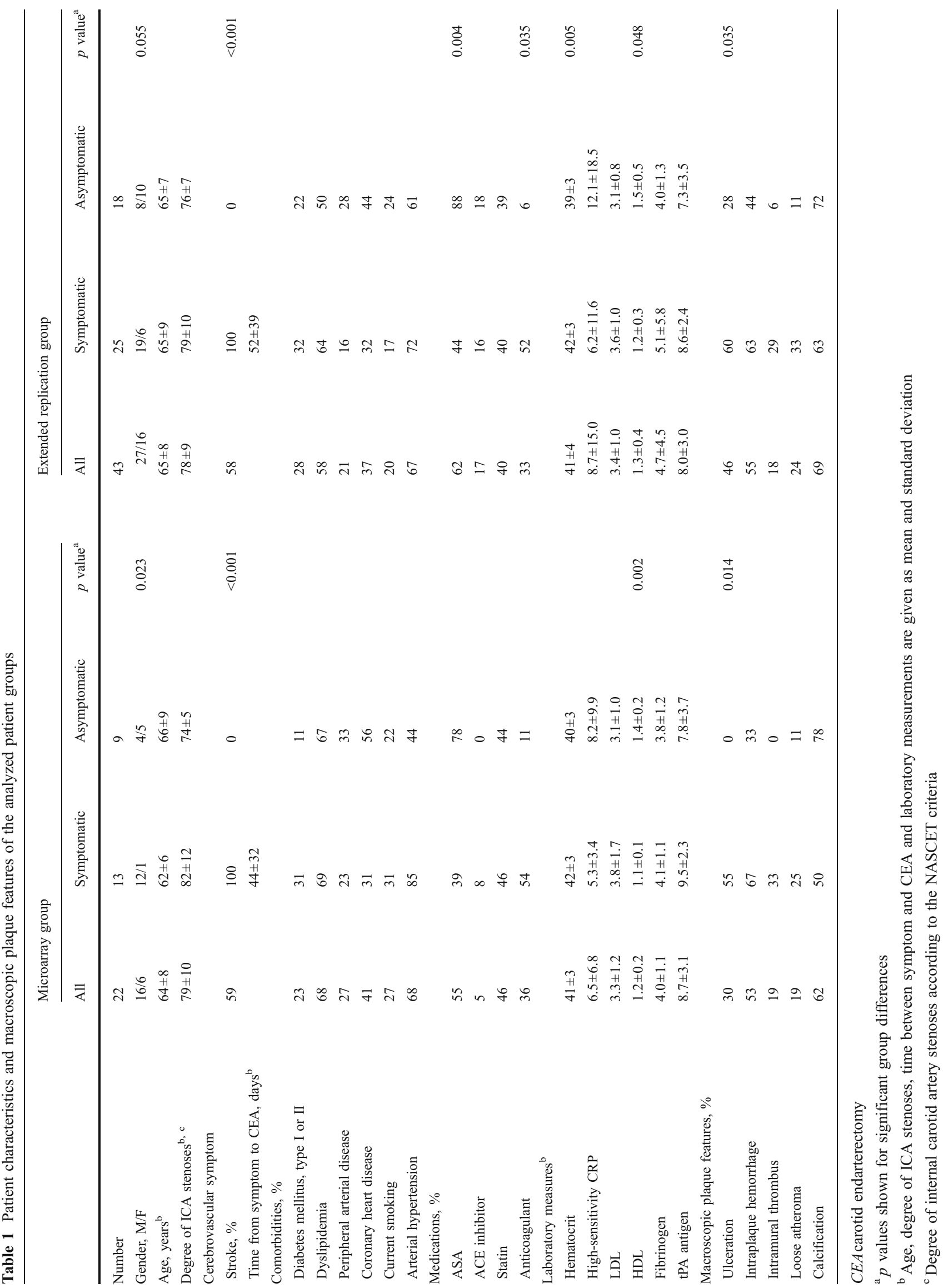


Fig. 1 Microarray data analysis flowchart. The number of probe sets remaining after each analysis step is shown. Dashed line arrows to the right indicate probe sets that were used for different clustering analysis. RMA Robust Multi-array Average. Asterisk (*) listed in Supplemental Table 3
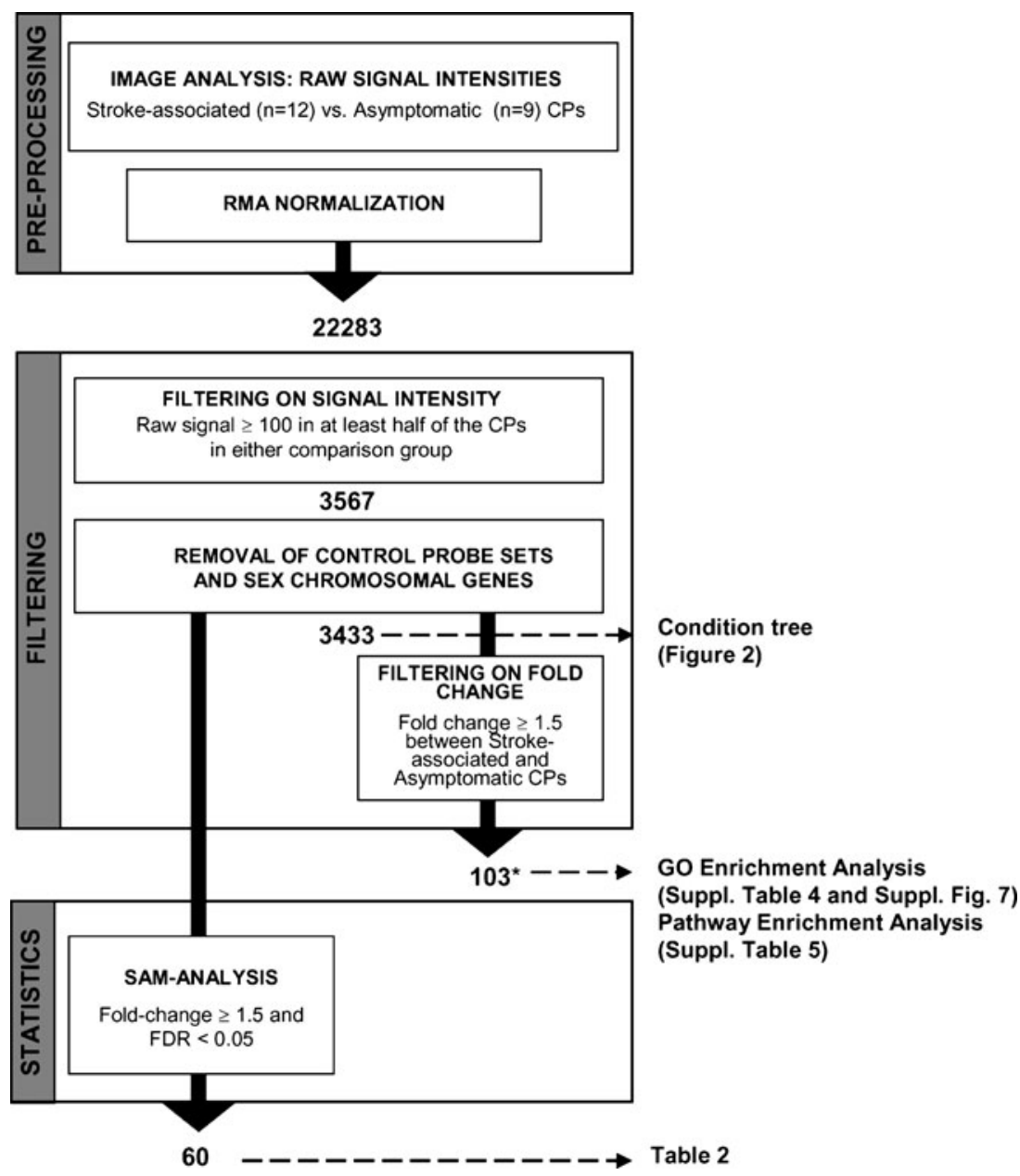

\section{Microarray analysis}

Figure 1 shows the flowchart of microarray analysis. Firstly, we employed hierarchical clustering of all the CPs and reliably expressed genes $(n=3,433$, see Fig. 1) to examine the relationships between CPs. The resulting Condition tree (Fig. 2) shows two main branches, one including ten of the 12 SCPs and the second, six of the nine

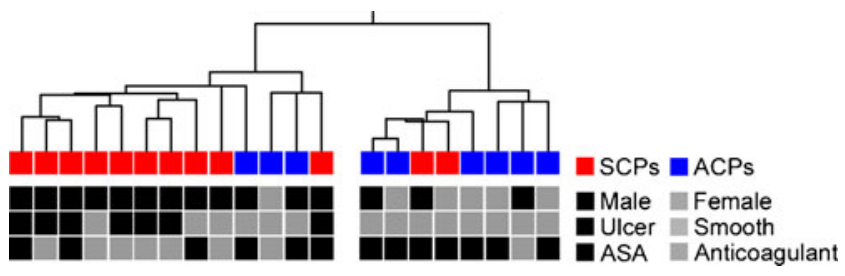

Fig. 2 Hierarchical clustering of CPs (Condition tree). The samples were classified into a tree based on their similarities. The length of the branch between two samples indicates how correlated their expression profiles are. The boxes below represent samples. The characteristics that were unevenly distributed between symptomatic and asymptomatic groups are shown
ACPs. Thus, the global gene expression profiles can classify $\mathrm{CPs}$ according to the symptom to asymptomatic and stroke-associated CPs. The classification accuracy remains the same, if clustering is performed for all genes ( $n=22,283$, see Fig. 1).

Ninety-two reliably expressed genes (103 probe sets) showed $\geq 1.5$-fold expression difference between strokeassociated and asymptomatic CPs (Supplemental Table 3). SAM analysis [21] of the 103 probe sets showed that 60 probes sets were significantly differentially expressed at $5 \%$ false discovery rate (Table 2). Since microarray analysis was shown to be sensitive to the choice of preprocessing algorithm [22], we analyzed the hybridization data also by two other commonly used algorithms, GC-RMA [23], and MAS5 (http://www.affymetrix.com), followed by comparable filtering steps (see Supplemental Material for details). Based on SAM analysis, 77 and 75 probe sets were significant at 5\% FDR in GC-RMA and MAS5 normalized data, respectively. Thirty-seven were shared by all three normalization methods (Supplemental Figure 6). 
Table 2 Microarray probe sets showing statistically significant expression difference between stroke-associated and asymptomatic carotid plaques

\begin{tabular}{|c|c|c|c|c|c|}
\hline Probe ID & Gene symbol $^{\mathrm{a}}$ & Gene name ${ }^{\mathrm{a}}$ & Fold-change & Mann-Whitney $\mathrm{U} p^{\mathrm{b}}$ & SAM q $(\%)^{b}$ \\
\hline 202206_at & ARL4C & ADP-ribosylation factor-like $4 \mathrm{C}$ & 1.6 & 0.001 & 1.72 \\
\hline 213418_at & HSPA6 & Heat shock $70 \mathrm{kDa}$ protein 6 & 1.8 & 0.001 & 1.72 \\
\hline 221760_at & MAN1A1 & Mannosidase, alpha, class $1 \mathrm{~A}$, member 1 & 1.5 & 0.002 & 1.72 \\
\hline 210512_s_at & VEGFA & Vascular endothelial growth factor A & 2.0 & 0.003 & 1.72 \\
\hline 200921_s_at & BTG1 & B-cell translocation gene 1, anti-proliferative & 1.6 & 0.004 & 1.72 \\
\hline 202207_at & ARL4C & ADP-ribosylation factor-like $4 \mathrm{C}$ & 1.6 & 0.004 & 1.72 \\
\hline 212192_at & KCTD12 & Potassium channel tetramerisation domain containing 12 & 1.6 & 0.004 & 1.72 \\
\hline 201670_s_at & MARCKS & Myristoylated alanine-rich protein kinase $\mathrm{C}$ substrate & 1.6 & 0.006 & 1.72 \\
\hline 202998_s_at & LOXL2 & Lysyl oxidase-like 2 & 1.7 & 0.006 & 1.72 \\
\hline 218149_s_at & ZNF395 & Zinc finger protein 395 & 1.5 & 0.006 & 1.72 \\
\hline 202499_s_at & SLC2A3 & Solute carrier family 2 , member 3 & 1.7 & 0.009 & 1.72 \\
\hline 205099_s_at & CCR1 & Chemokine $(\mathrm{C}-\mathrm{C}$ motif) receptor 1 & 1.6 & 0.009 & 1.72 \\
\hline 212154_at & SDC2 & Syndecan 2 & 1.8 & 0.009 & 1.72 \\
\hline 217028_at & CXCR4 & Chemokine ( $\mathrm{C}-\mathrm{X}-\mathrm{C}$ motif) receptor 4 & 1.8 & 0.009 & 1.72 \\
\hline 202310_s_at & COL1A1 & Collagen, type I, alpha 1 & 1.7 & 0.011 & 1.72 \\
\hline 212820_at & DMXL2 & DMX-like 2 & 1.7 & 0.011 & 1.72 \\
\hline 201438_at & COL6A3 & Collagen, type VI, alpha 3 & 1.7 & 0.013 & 1.72 \\
\hline 221210_s_at & NPL & $N$-acetylneuraminate pyruvate lyase & 1.8 & 0.013 & 1.72 \\
\hline 201193_at & IDH1 & Isocitrate dehydrogenase $1(\mathrm{NADP}+)$, soluble & 1.6 & 0.016 & 1.72 \\
\hline 202934_at & HK2 & Hexokinase 2 & 1.7 & 0.016 & 1.72 \\
\hline 213655_at & YWHAE & Tyrosine 3-monooxygenase & 1.6 & 0.016 & 1.72 \\
\hline 215049_x_at & CD163 & CD163 molecule & 1.6 & 0.016 & 1.72 \\
\hline 217983_s_at & RNASET2 & Ribonuclease T2 & 1.7 & 0.016 & 1.72 \\
\hline 32128_at & CCL18 & Chemokine (C-C motif) ligand 18 & 2.2 & 0.016 & 1.72 \\
\hline 203645_s_at & CD163 & CD163 molecule & 1.7 & 0.019 & 1.72 \\
\hline 209924_at & CCL18 & Chemokine (C-C motif) ligand 18 & 2.0 & 0.019 & 1.72 \\
\hline 212671_s_at & $\begin{array}{l}\text { HLA-DQA1/ } \\
\text { HLA-DQA2/ } \\
\text { LOC650946 }\end{array}$ & Major histocompatibility complex, class II DQ alpha 1 & 1.8 & 0.019 & 1.72 \\
\hline 201645_at & $\mathrm{TNC}$ & Tenascin $\mathrm{C}$ & 1.6 & 0.023 & 1.72 \\
\hline 202838_at & FUCA1 & Fucosidase, alpha-L-1 & 1.9 & 0.023 & 1.72 \\
\hline 212582_at & OSBPL8 & Oxysterol binding protein-like 8 & 1.6 & 0.023 & 1.72 \\
\hline 203814_s_at & NQO2 & $\mathrm{NAD}(\mathrm{P}) \mathrm{H}$ dehydrogenase, quinone 2 & 1.5 & 0.028 & 1.72 \\
\hline 217202_s_at & GLUL & Glutamate-ammonia ligase & 1.7 & 0.028 & 1.72 \\
\hline 200648_s_at & GLUL & Glutamate-ammonia ligase & 1.9 & 0.033 & 1.72 \\
\hline 202087_s_at & CTSL & Cathepsin L & 1.5 & 0.033 & 1.72 \\
\hline 202436_s_at & CYP1B1 & Cytochrome P450, family 1 , subfamily B, polypeptide 1 & 1.6 & 0.033 & 1.72 \\
\hline 202859_x_at & IL8 & Interleukin 8 & 1.9 & 0.033 & 1.72 \\
\hline 202902_s_at & CTSS & Cathepsin S & 1.5 & 0.033 & 1.72 \\
\hline 204438_at & MRC1/MRC1L1 & Mannose receptor, $\mathrm{C}$ type 1 & 1.6 & 0.033 & 1.72 \\
\hline 208146_s_at & CPVL & Carboxypeptidase, vitellogenic like & 1.5 & 0.036 & 1.72 \\
\hline 201147_s_at & TIMP3 & TIMP metallopeptidase inhibitor 3 & 1.9 & 0.039 & 1.72 \\
\hline 202345_s_at & FABP5 & Fatty acid-binding protein 5 & 1.6 & 0.047 & 1.72 \\
\hline 206488_s_at & CD36 & CD36 molecule & 2.2 & 0.047 & 1.72 \\
\hline 219607_s_at & MS4A4A & Membrane-spanning 4-domains, subfamily A, member 4 & 1.8 & 0.047 & 1.72 \\
\hline 202912_at & $\mathrm{ADM}$ & Adrenomedullin & 1.5 & 0.055 & 1.72 \\
\hline 203665_at & HMOX1 & Heme oxygenase 1 & 2.2 & 0.055 & 1.72 \\
\hline 203980_at & FABP4 & Fatty acid-binding protein 4 & 2.6 & 0.055 & 1.72 \\
\hline 212657_s_at & IL1RN & Interleukin 1 receptor antagonist & 1.6 & 0.055 & 1.72 \\
\hline
\end{tabular}


Table 2 (continued)

\begin{tabular}{|c|c|c|c|c|c|}
\hline Probe ID & Gene symbol $^{\mathrm{a}}$ & Gene name ${ }^{a}$ & Fold-change & Mann-Whitney $\mathrm{U} p^{\mathrm{b}}$ & SAM q $(\%)^{b}$ \\
\hline 214038_at & CCL8 & Chemokine $(\mathrm{C}-\mathrm{C}$ motif) ligand 8 & 1.6 & 0.055 & 1.72 \\
\hline 215223_s_at & SOD2 & Superoxide dismutase 2 , mitochondrial & 1.5 & 0.055 & 1.72 \\
\hline 204580_at & MMP12 & Matrix metallopeptidase 12 & 2.3 & 0.065 & 1.72 \\
\hline 209122_at & PLIN2 & Perilipin 2 & 1.8 & 0.065 & 1.72 \\
\hline 217294_s_at & ENO1 & Enolase 1 & 1.7 & 0.065 & 1.72 \\
\hline 200832_s_at & $\mathrm{SCD}$ & Stearoyl-CoA desaturase & 1.6 & 0.076 & 1.72 \\
\hline 209351_at & KRT14 & Keratin 14 & 1.8 & 0.076 & 1.72 \\
\hline 209555_s_at & CD36 & CD36 molecule & 1.9 & 0.076 & 1.72 \\
\hline 201785_at & RNASE1 & Ribonuclease, RNase A family, 1 & 1.5 & 0.118 & 3.26 \\
\hline 202917_s_at & S100A8 & S100 calcium binding protein A8 & 1.6 & 0.118 & 3.26 \\
\hline 221730_at & COL5A2 & Collagen, type V, alpha 2 & 1.5 & 0.136 & 3.26 \\
\hline 203381_s_at & APOE & Apolipoprotein E & 1.6 & 0.177 & 4.00 \\
\hline 204259_at & MMP7 & Matrix metallopeptidase 7 & 1.5 & 0.201 & 4.72 \\
\hline
\end{tabular}

${ }^{a}$ For the probe set detecting transcripts from several homologous genes, symbols are separated by hyphen. Gene name is only given for the first one

${ }^{\mathrm{b}} p=$ pairwise non-adjusted $p$ values from Mann-Whitney $U$ test, $\mathrm{q}=$ the minimum false discovery rate at which the gene is called significant from significance analysis of microarrays (SAM)

To identify pathophysiologically important gene groups, we performed gene enrichment analysis on Gene Ontology (GO) categories and Kyoto Encyclopedia of Genes and Genomes (KEGG) and Biocharta pathways for the differentially expressed genes ( $n=103$, Supplemental Table 3$)$. Fifty-one GO categories showed enrichment with the most significant $(p<0.001)$ categories: extracellular matrix, extracellular space, lysosome, lytic vacuole, vacuole, fibrillar collagen, collagen, scavenger receptor activity, peptidoglycan metabolism, response to wounding and organ development (categories with $\geq 3$ genes and $p<0.01$ are shown in the Supplemental Figure 7 and Supplemental Table 4). Six KEGG and one Biocharta pathway showed more than two differentially expressed genes, and the most significant pathways $(p<0.001$, in order of significance) were the extracellular matrix-receptor interaction, PPAR signaling, and antigen processing and presentation pathways (Supplemental Table 5).

Confirmation of differentially expressed genes

in the extended replication group

Differential expression of 18 genes discovered in the microarray experiment was tested by qPCR in the same samples and congruent fold change was found for 16 genes (89\%, Fig. 3). Next we analyzed these 18 genes in the extended replication group $(n=43)$, which included CPs from clinically defined stroke patients $(n=25)$ and asymptomatic patients $(n=18)$, and qPCR confirmed significant differential expression of ten genes between SCPs and ACPs (Fig. 3). Confirmed expression changes with the most significant association $(p<0.01)$ with symptoms were
CD36 (2.1-fold change, $p=0.005)$ and CD163 (1.7-fold change, $p=0.007)$.

Correlations of differentially expressed genes with clinical data and plaque features

We investigated whether the ten genes with confirmed expression changes showed any association with clinical patient characteristics or morphological plaque features (Supplemental Table 6). After multiple testing correction, we did not find significant associations to known atherosclerosis risk factors, comorbidities, medications, or plaque cell-type markers. Instead, several genes showed association to plaque ulceration (seven out of ten genes), intraplaque hemorrhage (four out of ten), and markers of cell death and proliferation (activated caspase 3, four out of ten; Ki67 proliferation marker, four out of ten; TUNEL positivity in the intima, two out of ten). Additionally, three genes showed positive correlation to the degree of ICA stenosis.

Immunohistochemical verification of proteins encoded by differentially expressed genes

The expression and histological localization of nine genes (CD36, CD163, FABP4, PLIN2, GLUL, CCL18, IL1RN, HMOX1, and S100A8) with qPCR-confirmed differential expression in SCPs were investigated at the protein level using immunohistochemistry (Fig. 4). CD36, CD163, FABP4, PLIN2, HMOX1, GLUL, and S100A8 showed expression mainly in macrophages. HMOX1, GLUL, S100A8, and IL1RN were also expressed in other cell 


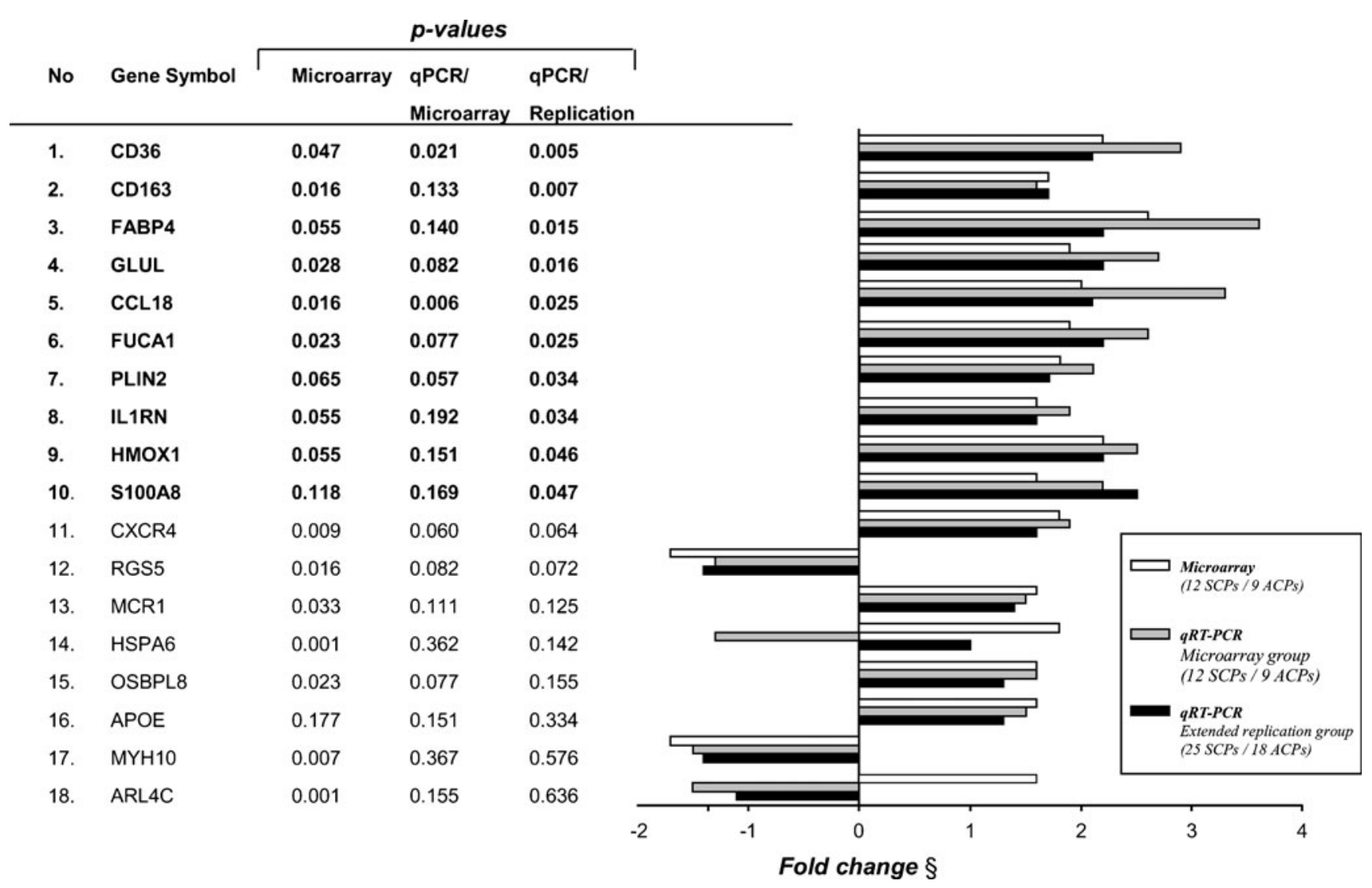

Fig. 3 Confirmation of differential gene expression by qPCR. Genes are organized according to statistical significance (significant bolded). Microarray refers to non-adjusted $p$ values from microarray analysis also given in Table 2 and Supplemental Table 3. QPCR/microarray

types as judged by morphology: HMOX1, GLUL, and IL1RN in smooth muscle cells and S100A8 in scarce intimal polymorphonuclear cells. IL1RN was also expressed in endothelial cells, both in the neovessels as well as in the luminal endothelium. CCL18 showed a diffuse intimal staining pattern typical for secreted chemokines. CD36, CD163, FABP4, PLIN2, S100A8, HMOX1, and GLUL showed strong staining around the lipid core and in atheromatous areas.

\section{Discussion}

The evolution of an atherosclerotic $\mathrm{CP}$ into an unstable plaque leading to atherothromboembolic strokes, is still incompletely understood. We adopted large-scale microarray analysis to identify genes and cellular pathways that could relate to plaque destabilization. Microarray analysis was focused on two phenotypic extremes: symptomatic CPs from patients with recent ipsilateral ischemic brain infarct and asymptomatic CPs from patients that have never experienced cerebrovascular symptoms and had normal refers to the real-time qPCR analysis results in the microarray group and $q P C R /$ replication in the extended replication group. All $p$ values from Mann-Whitney $U$ test

brain imaging. We demonstrate that stroke-associated CPs possess a characteristic gene expression profile, which distinguishes them from asymptomatic CPs. In addition to confirming several previously recognized genes linked to unstable atherosclerosis, our analysis revealed expression changes in genes with no previously known association with symptomatic or unstable atherosclerotic plaques. Functional clustering data imply intrinsic differences between ACPs and SCPs in cellular processes, including inflammatory responses, intracellular lipid and carbohydrate metabolism and transport, lysosomal activity, and reorganization of the extracellular matrix.

The microarray analysis revealed differential expression with more than 1.5 -fold change in 103 probe sets representing 92 genes. SAM analysis of these 103 probe sets found 60 probe sets to be statistically significant at $5 \%$ FDR (Fig. 1 and Table 2). This represents approximately

Fig. 4 Immunohistochemical stainings of CD36, CD163, PLIN2, FABP4, GLUL, CCL18, IL1RN, HMOX1, and S100A8. The first column displays images obtained from the edge of an atheroma. The second and the third columns are magnifications from foam cell-rich areas indicated by rectangles 


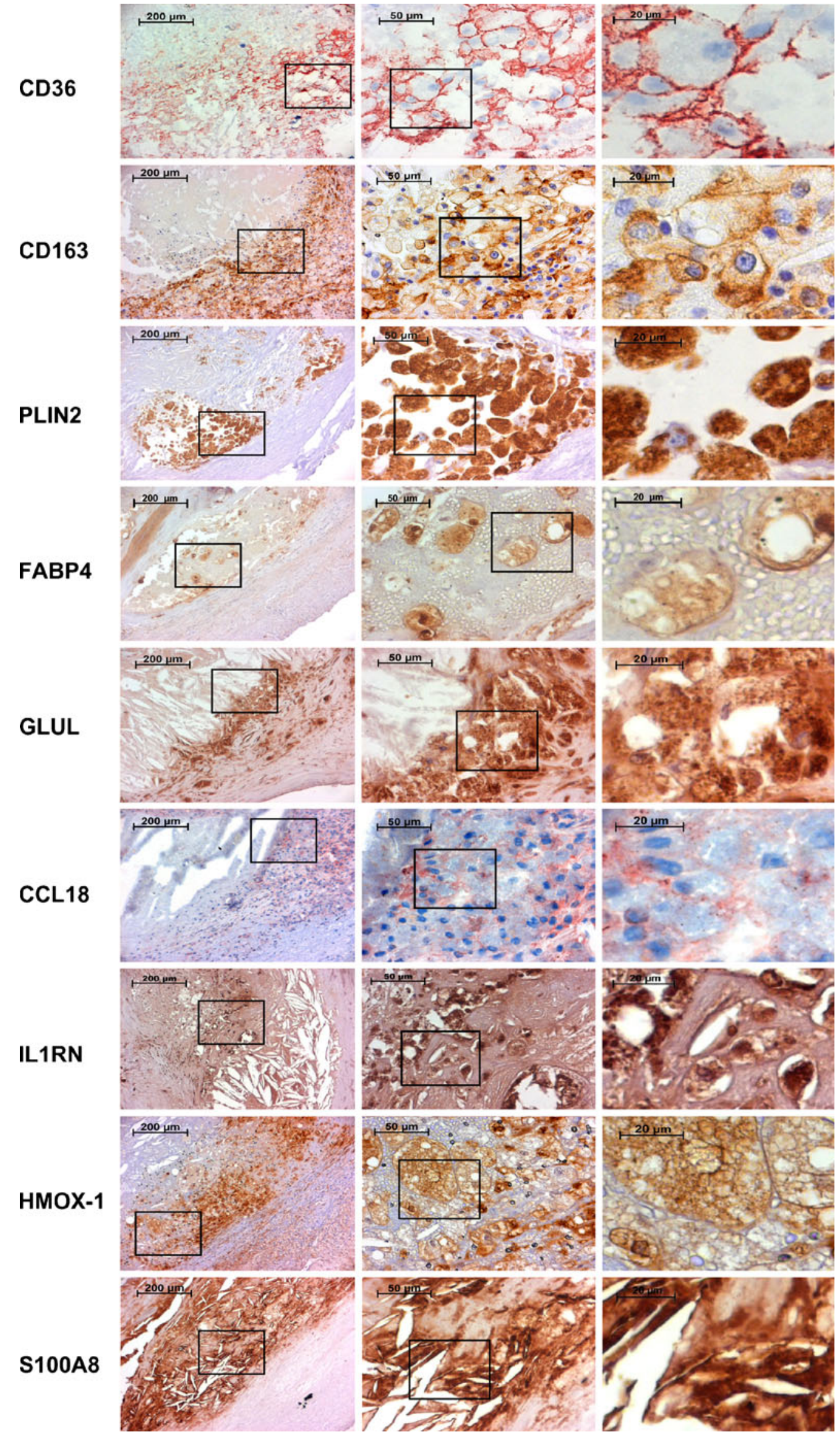


$0.3 \%$ of the probe sets in the original microarray. A small number of differentially expressed genes are expected, since we compare samples that all represent advanced atherosclerotic tissue with no clear histological differences. In addition, we used a stringent filtering criteria and accepted $15 \%$ of the microarray probe sets for statistical analysis. The decision on this high filtering cutoff was based on a fact that in a heterogenous tissue such as atherosclerotic plaque, any expression signal measured is a sum of signals from numerous cell populations. Typically, this leads to low fold changes, when only a proportion of cells express a given gene or different cell population show reverse expression changes overriding each others' effect. In this kind of situation, detection of low fold "true" changes from background fluctuation is difficult. Therefore, we chose to concentrate on transcripts whose expression level and difference are high enough to allow for their reliable detection with a goal of finding reproducible list of differentially expressed genes, even if we recognize that this probably increases type II errors.

Eighteen genes were selected for replication by qPCR both in the microarray group and in the extended replication group. Several criteria were used in the selection: statistical significance, consistency across preprocessing methods, functional clustering analysis, and novelty. Replication rate was $89 \%$ (16 out of 18 genes) in the microarray group. Only ARL4C and HSPA6 failed to show differential expression by qPCR despite being the two most significant genes in the microarray analysis. Both genes have only one exon, which might cause problems in the correct measurement of single-exon genes by the array's probe design. Findings were similar in the extended replication group: all except ARL4C and HSPA6 showed congruent fold changes in magnitude and direction (Fig. 3). The expression change was statistically significant for ten of the 18 genes (56\%). We confirmed protein expression of these genes in the CPs by immunohistochemistry and show that they are expressed in the atheromatous areas of CPs critical for plaque destabilization (Fig. 4).

Many of the identified genes show correlation to plaque features that can be considered markers of CP instability, namely ulceration [5, 7], intraplaque hemorrhage [5], and apoptosis [24] (Supplemental Table 6). In line with the finding of the higher frequency of macroscopic ulcerations in our stroke-associated CPs (see Table 1), the expression of $80 \%$ of the qPCR-confirmed genes showed correlation to macroscopic ulcerations. Plaque ulceration is an integral phase in the development of sudden thromboembolism and in this way a morphological correlate of plaque instability [7, 25-27]. It is likely that ulceration affects gene expression patterns and may partly explain them, i.e., through the process of wound healing. However, ulceration and thromboembolism are not "one-time events" but rather the unstable plaques have a chronic course of disease with repeated thromboembolic events. For example, 36\% of patients in our extended replication group had TIA within 6 months prior to ischemic stroke and NASCET showed that without CEA the risk of new event stays high several months until it drops gradually within 2 to 3 years to the same annual $3 \%$ level as found in asymptomatic patients [1]. It is possible that the recurrent cycles of rupture and healing with the concomitant ulceration of endothelial lining and intraplaque hemorrhages are integral to the instability of symptomatic plaques.

Seven of the qPCR-replicated genes also showed correlation to intraplaque hemorrhages (IPH). In the large meta-analysis of Gao and colleagues [28], incidence of IPH was higher in symptomatic plaques, but the evidence mainly derived from early small-sized studies, whereas recent larger studies yielded insignificant associations. We did not find difference in the incidence of IPH between ACPS and SCPS in our study groups or in the whole HeCES cohort (Table 1) [17]. However, these observations do not exclude the role of IPH in plaque destabilization. In patients with asymptomatic moderate carotid stenosis, MRI-depicted IPH was associated with future ipsilateral cerebrovascular events [27]. IPH or marked intraplaque vessel formation were also shown to be the only plaque features to predict cardiovascular events after CEA [29]. IPH can destabilize plaques both by ensuing oxidative and inflammatory stress as well as exacerbating the local lipid burden by entailing red blood cell membranes abundant in cholesterol $[14,20,30,31]$. Our own data suggests that IPH lead to a more-pronounced inflammatory gene induction in symptomatic than in asymptomatic CPs, which could be explained by differences in genetic factors or plaque microenvironment [14]. This kind of phenomenon could explain ambiguous results from previous observational studies.

The results obtained from the present study concur well with the results from our earlier intra-patient expression analysis [14] (15/92 (16\%) of the differentially expressed genes) as well as with the results from Papaspyridonos and colleagues comparing unstable and stable plaque regions [9] (33/92 (36\%) of the differentially expressed genes). Both matrix metallopeptidase 9 and legumain reported by Papaspyridonos and colleagues [9] are significantly overexpressed in the SCPs of our material. Instead, we do not find increased expression of cathepsin B, but cathepsins D, $\mathrm{L}$, and $\mathrm{S}$. We detect the strong over-expression of FABP4 in the stroke-associated $\mathrm{CPs}$, as was recently reported for symptomatic CPs by two groups [11, 32]. Increased expression of visfatin is not found in our material, but 20 of the genes showing differential expression (although not reaching statistical significance) in the microarray study by Dahl and colleagues [10], were also differentially expressed 
in our stroke-associated CPs. Converging evidence from these studies accumulates for the important role of several genes in the destabilization of CPs, especially cathepsin L, CD36, fatty acid-binding proteins 4 and 5 (FABP4 and FABP5), heme oxygenase 1, interleukin 1 receptor antagonist (IL1RN), interleukin 8, matrix metalloproteinases 7 and $12, \mathrm{~N}$-acetylneuraminate pyruvate lyase (NPL), nonmuscle myosin 10 (MYH10) and perilipin 2 (PLIN2, previously known as ADFP).

There is a significant difference between the proportion of women in the symptomatic and asymptomatic groups (Table 1). Skewed gender distribution is an inherent epidemiological phenomenon incorporated in all consecutive series of symptomatic carotid disease [1, 2]. In this study, the gender difference is partly due to the fact that the microarray analysis was restricted to the phenotypic extremes of the disease defined by strict clinical and radiological criteria and hence constituted a subgroup of the larger gender balanced HeCES cohort. To partially compensate for the gender imbalance, genes located in sex chromosomes were excluded from the microarray data analysis. Despite of this imbalance, RNA expression levels of only three of the 18 genes replicated by qPCR showed any correlation to gender (Supplemental Table 6). Also, hierarchical clustering of samples based on global gene expression patterns show that symptom and macroscopic ulcerations are stronger determining factors of gene expression than gender (Fig. 2).

In this study, we report a comprehensive transcriptional analysis of CPs from individuals with clinically relevant carotid disease that links plaque-level gene expression data to thromboembolic strokes. Since the assignment to symptom groups was performed purely from a clinical standpoint, we consider the reported transcriptome to genuinely represent clinical vulnerability of CPs. Perhaps the most striking finding of the present study is that the resulting transcriptome clearly differentiates strokeassociated CPs from asymptomatic CPs at the level of individual patients, corresponding to the clinical course (Fig. 2). Unlike many systemic markers analyzed in our patient material, the plaque-level analysis revealed significant differences related to carotid plaque behavior. This indicates that local expression changes within the plaque might be more crucial in the longstanding development of plaque vulnerability than incidental systemic factors that might have a more transient triggering role in acute plaque destabilization. These results suggest potential new pharmacological targets to be explored for the management, stabilization, or treatment of unstable large artery atherosclerosis.

Acknowledgments Taru Puhakka, Saija Eirola, and Riitta Kärkkäinen are thanked for their skillful technical assistance. This study was funded by Helsinki University Central Hospital (EVO) research grants, the Academy of Finland and the Sigrid Jusélius, Lundbeck, Aarne Koskelo, Maire Taponen, and Päivikki and Sakari Sohlberg foundations. Wihuri
Research Institute is maintained by the Jenny and Antti Wihuri foundation.

Disclosures None.

Open Access This article is distributed under the terms of the Creative Commons Attribution Noncommercial License which permits any noncommercial use, distribution, and reproduction in any medium, provided the original author(s) and source are credited.

\section{References}

1. NASCET collaborators (1991) North American Symptomatic Carotid Endarteroctomy Trial. Beneficial effect of carotid endarterectomy in symptomatic patients with high-grade carotid stenosis. N Engl J Med 325:445-453

2. No author (1998) Randomised trial of endarterectomy for recently symptomatic carotid stenosis: final results of the MRC European Carotid Surgery Trial (ECST). Lancet 351:1379-1387

3. Golledge JM, Greenhalgh RM, Davies AH (2000) The symptomatic carotid plaque. Stroke 31:774-781

4. Virmani R, Burke AP, Farb A, Kolodgie FD (2002) Pathology of the unstable plaque. Prog Cardiovasc Dis 44:349-356

5. Redgrave JN, Lovett JK, Gallagher PJ, Rothwell PM (2006) Histological assessment of 526 symptomatic carotid plaques in relation to the nature and timing of ischemic symptoms: The Oxford Plaque Study. Circulation 113:2320-2328

6. Peeters W, Hellings WE, de Kleijn DP, de Vries JP, Moll FL, Vink A, Pasterkamp G (2009) Carotid atherosclerotic plaques stabilize after stroke. Insights into the natural process of atherosclerotic plaque destabilization. Arterioscler Thromb Vasc Biol 29:128-133

7. Eliasziw M, Streifler JY, Fox AJ, Hachinski VC, Ferguson GG, Barnett HJ (1994) Significance of plaque ulceration in symptomatic patients with high-grade carotid stenosis. Stroke 25:304-308

8. Vemuganti R, Dempsey RJ (2005) Carotid atherosclerotic plaques from symptomatic stroke patients share the molecular fingerprints to develop in a neoplastic fashion: a microarray analysis study. Neuroscience 131:359-374

9. Papaspyridonos M, Smith A, Burnand KG, Taylor P, Padayachee P, Suckling KE, James CH, Greaves DR, Patel L (2006) Novel candidate genes in unstable areas of human atherosclerotic plaques. Arterioscler Thromb Vasc Biol 26:1837-1844

10. Dahl TB, Yndestad A, Skjelland M, Oie E, Dahl A, Michelsen A, Damas JK, Tunheim SH, Ueland T, Smith C et al (2007) Increased expression of visfatin in macrophages of human unstable carotid and coronary atherosclerosis: possible role in inflammation and plaque destabilization. Circulation 115:972-980

11. Agardh HE, Folkersen L, Ekstrand J, Marcus D, Swedenborg J, Hedin U, Gabrielsen A, Paulsson-Berne G (2010) Expression of fatty acid-binding protein $4 / \mathrm{aP} 2$ is correlated with plaque instability in carotid atherosclerosis. J Intern Med 269:200-210

12. Slevin M, Elasbali AB, Miguel Turu M, Krupinski J, Badimon L, Gaffney J (2006) Identification of differential protein expression associated with development of unstable human carotid plaques. Am J Pathol 168:1004-1021

13. Lepedda AJ, Cigliano A, Cherchi GM, Spirito R, Maggioni M, Carta F, Turrini F, Edelstein C, Scanu AM, Formato M (2009) A proteomic approach to differentiate histologically classified stable and unstable plaques from human carotid arteries. Atherosclerosis 203:112-118

14. Ijäs P, Nuotio K, Saksi J, Lehtonen-Smeds EM, Soinne L, Saimanen E, Karjalainen-Lindsberg M, Salonen O, Sarna S, 
Tuimala J et al (2007) Microarray analysis reveals over-expression of CD163 and HO-1 in symptomatic carotid plaques. Arterioscler Thromb Vasc Biol 27:154-160

15. Nuotio K, Lindsberg PJ, Carpén O, Soinne L, Lehtonen-Smeds EM, Saimanen E, Lassila R, Sairanen T, Sarna S, Salonen O et al (2003) Adhesion molecule expression in symptomatic and asymptomatic carotid stenosis. Neurology 60:1890-1899

16. Lehtonen-Smeds EM, Mäyränpää M, Lindsberg PJ, Soinne L, Saimanen E, Jarvinen AA, Salonen O, Carpen O, Lassila R, Sarna $\mathrm{S}$ et al (2005) Carotid plaque mast cells associate with atherogenic serum lipids, high grade carotid stenosis and symptomatic carotid artery disease - results from the Helsinki Carotid Endarterectomy Study. Cerebrovasc Dis 19:291-301

17. Soinne L, Saimanen E, Malmberg-Ceder K, Kovanen P, Lindsberg PJ, Kaste M, Lassila R (2005) Association of the fibrinolytic system and hemorheology with symptoms in patients with carotid occlusive disease. Cerebrovasc Dis 20:172-179

18. Brazma A, Hingamp P, Quackenbush J, Sherlock G, Spellman P, Stoeckert C, Aach J, Ansorge W, Ball CA, Causton HC et al (2001) Minimum information about a microarray experiment (MIAME) toward standards for microarray data. Nat Genet 29:365-371

19. Stary H, Chandler A, Dinsmore R, Fuster V, Glagov S, Insull W, Rosenfeld M, Schwartz C, Wagner W, Wissler R (1995) A definition of advanced types of atherosclerotic lesions and a histological classification of atherosclerosis: a report from the Committee on Vascular Lesions of the Council on Arteriosclerosis, American Heart Association. Circulation 92:1355-1374

20. Nuotio K, Isoviita PM, Saksi J, Pitkämäki J, Ijäs P, Soinne L, Saimanen E, Salonen O, Kovanen PT, Kaste M et al (2007) Adipophilin expression is increased in symptomatic carotid atherosclerosis and co-localizes with red blood cells and cholesterol crystals. Stroke 38:1791-1798

21. Tusher VG, Tibshirani R, Chu G (2001) Significance analysis of microarrays applied to the ionizing radiation response. Proc Natl Acad Sci USA 98:5116-5121

22. Irizarry RA, Wu Z, Jaffee HA (2006) Comparison of Affymetrix GeneChip expression measures. Bioinformatics 22:789-794

23. Wu Z, Irizarry RA (2004) Preprocessing of oligonucleotide array data. Nat Biotech 22:656-658
24. Geng YJ, Libby P (2002) Progression of atheroma: a struggle between death and procreation. Arterioscler Thromb Vasc Biol 22:1370-1380

25. Sitzer M, Muller W, Siebler M, Hort W, Kniemeyer H, Jancke L, Steinmetz H (1995) Plaque ulceration and lumen thrombus are the main sources of cerebral microemboli in high-grade internal carotid artery stenosis. Stroke 26:1231-1233

26. Rothwell PM, Gibson R, Warlow CP (2000) Interrelation between plaque surface morphology and degree of stenosis on carotid angiograms and the risk of ischemic stroke in patients with symptomatic carotid stenosis. On behalf of the European Carotid Surgery Trialists' Collaborative Group. Stroke 31:615-621

27. Takaya N, Yuan C, Chu B, Saam T, Underhill H, Cai J, Tran N, Polissar NL, Isaac C, Ferguson MS et al (2006) Association between carotid plaque characteristics and subsequent ischemic cerebrovascular events: a prospective assessment with MRI-initial results. Stroke 37:818-823

28. Gao P, Chen Z, Bao Y, Jiao L, Ling F (2007) Correlation between carotid intraplaque hemorrhage and clinical symptoms-systematic review of observational studies. Stroke 38:2382-2390

29. Hellings WE, Peeters W, Moll FL, Piers SR, van Setten J, Van der Spek PJ, de Vries JP, Seldenrijk KA, De Bruin PC, Vink A, Velema E, de Kleijn DP, Pasterkamp G (2010) Composition of carotid atherosclerotic plaque is associated with cardiovascular outcome: a prognostic study. Circulation 121:1941-50

30. Isoviita PM, Nuotio K, Saksi J, Turunen R, Ijäs P, Pitkäniemi J, Soinne L, Kaste M, Kovanen PT, Lindsberg PJ (2010) An imbalance between $\mathrm{CD} 36$ and $\mathrm{ABCA} 1$ protein expression favors lipid accumulation in stroke-prone ulcerated carotid plaques. Stroke 2:389-393

31. Michel JB, Virmani R, Asrbustini E, Pasterkamp G (2011) Intraplaque haemorrhages as the trigger of plaque vulnerability. Eur Heart J. doi:10.1093/eurheartj/ehr054

32. Peeters W, de Kleijn DP, Vink A, van de Weg S, Schoneveld AH, Sze SK, van der Spek PJ, de Vries JP, Moll FL, Pasterkamp G (2010) Adipocyte fatty acid binding protein in atherosclerotic plaques is associated with local vulnerability and is predictive for the occurrence of adverse cardiovascular events. Eur Heart J. doi:10.1093/eurheartj/ehq387 